\title{
GUEST EDITORIAL Questions about DISC1 as a genetic risk factor for schizophrenia
}

Molecular Psychiatry (2013) 18, 1050-1052; doi:10.1038/mp.2012.182

A welcome change in psychiatric genetics has been the widespread recognition of the essential role of uncompromising statistical rigor and replication. Put simply, the genome is a big place, and it is trivial to find false leads-non-significant but 'suggestive' genomic findings that an integrative scientist might find 'intriguing'.

Indeed, due to advances in sequencing technology, the next few years are certain to see an explosion in observations of unique events in people with schizophrenia and other psychiatric disorders. Some of these will be claimed to be causal. However, the paucity of results from exome sequencing of sizable samples in autism ${ }^{1-3}$ and schizophrenia, ${ }^{4,5}$ combined with the surprisingly high rates of deleterious exonic variation in apparently normal people, ${ }^{6}$ indicates that it will be highly challenging to delineate disease-related variants from background noise. For example, even with the improbably optimistic assumption that $1 \%$ of schizophrenia cases are caused by fully penetrant mutations in one gene that has no confounding background variation, observing 10 deleterious mutations in 1000 cases and 0 in 1000 controls would not be clearly delineated from the distribution of test statistics across 15-20000 genes. In reality, locus heterogeneity, incomplete penetrance and realistic background variation will make this task markedly more difficult.

As there is already an influential example of a unique genomic event, it is timely to review the genomics of 'Disrupted in Schizophrenia 1' (DISC1), a $\mathrm{t}(1 ; 11)$ (q42.1;q14.3) structural variant identified using cytogenetic methods. ${ }^{7,8}$ (The chromosomal bands are sometimes different from $1 \mathrm{q} 42.1$ and 11q14.3. I determined these bands by mapping the breakpoint sequences in Millar et al. ${ }^{7}$ to hg19 using UCSC/BLAT.) Over 20 years after the initial report, the status of DISC1 as a risk factor for schizophrenia is unclear and perhaps polarized: some researchers are convinced that it is a proven etiological factor in schizophrenia, and others that it is not Other groups await empirical data to resolve its role. Indeed, my group has found non-significant but 'intriguing' results about DISC1 twice, and both times its potential salience faded with more data. ${ }^{9,10}$

The purpose of this editorial is to review the genetic evidence for the involvement of DISC1 in schizophrenia. There are important unanswered questions that need to be resolved for DISC1 to be established as a bona fide genetic risk factor for schizophrenia.

\section{VIEWS ON DISC1 IN THE LITERATURE}

Some consider DISC1 as a proven risk factor for schizophrenia. ${ }^{11,12}$ Examples of statements about DISC1 include: 'this private mutation has revealed important mechanisms of disease', ${ }^{13}$ 'a key susceptibility gene for schizophrenia is $\mathrm{DISC1}^{\prime}$, ${ }^{14}$ 'a susceptibility gene for schizophrenia', ${ }^{15}$ 'a convincing candidate gene'16 and 'DISC1, a major susceptibility factor for several mental disorders'. ${ }^{17}$ Some psychiatric disorders have been termed 'DISC1opathies', ${ }^{18}$ and DISC1 has been referred to as the 'special gene'.

\section{THE DISC1 PEDIGREE}

The pedigree was initially reported in 1970, and identified via an 18-year-old male karyotyped in a cytogenetic study of boys sentenced to a youth prison in Scotland. ${ }^{19}$ The propositus had conduct disorder, and none of his first-degree relatives had a psychotic disorder.

Three cytogenetic abnormalities were reported to segregate in this pedigree: a balanced translocation between chr1 and a group C chromosome (chr6-12), a separate chr1 'unusually large secondary constriction', and a Robertsonian translocation between two group D chromosomes (chr13-15). To my knowledge, the most recent report of the phenotypes in the pedigree was in $2001,{ }^{20}$ but the 2001 pedigree is considerably smaller than that in the 1970 report. Diagnoses were established using a structured diagnostic interview by psychiatrists blinded to genotype, and of 29 individuals with $\mathrm{t}(1 ; 11)$ (q42.1;q14.3): 11 (37.9\%) had no diagnosis, an anxiety disorder, conduct disorder or alcohol dependence 10 (34.4\%) had recurrent major depressive disorder; and 8 (27.6\%) had a psychotic disorder (7 schizophrenia and 1 bipolar disorder). Parametric linkage analyses under a dominant model maximized at a logarithm of odds (LOD) score of 7.1 when recurrent major depressive disorder, schizophrenia and bipolar disorder were considered affected. The next largest LOD of 4.5 was for mood disorders (recurrent major depressive disorder and bipolar disorder), and schizophrenia alone had a LOD of 3.6.

These reports do not answer multiple questions of interest to the research community (Table 1$)$. First, it is possible that $t(1 ; 11)$ (q42.1; 14.3 ) status is based on laboratory assessments done over 40 years ago. This should give any researcher pause, particularly if the key linkage analyses in Blackwood et al. ${ }^{20}$ are based on the Jacobs et al. ${ }^{19}$ structural variant assignments. Second, I could find no published explanation or analysis of why the researchers focused on one of the three structural variants reported to segregate in this pedigree. Third, critically, sensitivity analyses were not reported (that is, systematically changing diagnoses within the pedigree and re-evaluating linkage evidence). The importance of these analyses was amply illustrated by the oldorder Amish linkage studies in the late 1980s, where a LOD of 4.9 faded to non-significance with a few changes in the pedigree. ${ }^{21}$ It is possible that the reported LOD scores are fragile and sensitive to changes in diagnostic status.

Fourth, the logical connections of $t(1 ; 11)$ ( $q 42.1 ; q 14.3)$ with schizophrenia are not compelling. The propositus and his immediate relatives have conduct disorder. The linkage analyses are more consonant with a mood disorder phenotype. The high prevalence of recurrent MDD is disconcerting given the predominant role of environmental risk factors in its etiology. ${ }^{22,23}$ Of greatest concern is that mental retardation, autism spectrum disorders and epilepsy have not been reported to segregate with $\mathrm{t}(1 ; 11)(\mathrm{q} 42.1 ; \mathrm{q} 14.3)$ in this pedigree. This is atypical for rare structural variants of strong effect that tend to increase the risk for multiple neuropsychiatric disorders. ${ }^{24}$

DISC1 proponents have argued that the lack of a uniform connection to a single psychiatric phenotype is expected and consistent with genetic risk factors having pleomorphic effects. Empirical data have suggested that pleomorphic effects are indeed the case ${ }^{.25}$ however, this does not appear to be a cleanly falsifiable argument in this pedigree. Indeed, if this argument were true, the authors make the case that 'disrupted in schizophrenia' is a misnomer.

\section{THE FOCUS ON THE CHR1 TRANSLOCATION REGION}

The $t(1 ; 11)$ ( $q 42.1 ; q 14.3)$ structural variant was identified as disrupting a novel gene that was given the name DISC1. 
Table 1. Unanswered questions about DISC1

The pedigree

Have the karyotypes from the late 1960 s been updated with modern methods? Were the key Blackwood et al. ${ }^{20}$ linkage analyses based on the Jacobs et al. ${ }^{19}$ karyotypes?

Three structural variants were reported to segregate in this pedigree: which can be verified with modern methods? Which segregate with psychiatric phenotypes? What was the justification for focusing solely on $t(1 ; 11)(q 42.1 ; q 14.3)$ ? Why was the rest of the pedigree not reported? The most recent phenotype reports are from 2001. How have the diagnoses changed? What effect do changes in diagnosis have on the linkage results? Given that linkage results can be sensitive to influential subjects, what do sensitivity analyses show?

The phenotypes that appear to track with $\mathrm{t}(1 ; 11)(\mathrm{q} 42.1 ; \mathrm{q} 14.3)$ are dissimilar to other rare structural variants where schizophrenia, autism, epilepsy and/or mental retardation are associated. The prominence of recurrent MDD is worrying. Why is this pedigree different? Does the absence of these other conditions suggest that DISC1 is not a true schizophrenia risk factor?

The focus on DISC1

It is possible that the chr1 DISC1 side of the breakpoint is not centrally important: what role does the chr11 side of the breakpoint play (for example, the predicted lincRNA)?

Much rests on the assumption that the translocation that impacts DISC1 is causal. However, efforts to falsify this genomic hypothesis are few. How can genomic data be used to more clearly implicate or exclude DISC1?

\section{Genetic results}

The DISC1 translocation is private to a single pedigree. The largest and most rigorously conducted genomic studies of common variation, rare variation and copy number variation provide no support for a role of DISC1 in schizophrenia, bipolar disorder, autism and MDD. A rigorous analysis of pleomorphic effects similarly found no evidence for a role for DISC1. Do these negatives exclude DISC1 with confidence?

Although this was a standard medical genetics approach, there are additional unanswered questions. First, the chr11 side of the breakpoint disrupts a predicted long intergenic non-coding RNA (lincRNA, ENST00000562245.1 or RP11-660M18.2). Such RNA molecules are expressed and do not code for protein, but can have important regulatory roles. Second, as noted above, $t(1 ; 11)$ $(q 42.1 ; q 14.3)$ is one of the three structural variants reported in this pedigree, and other structural variants could be relevant. Third, the members of this pedigree share considerable amounts of the genome identical-by-descent; have the relevance of other genetic variants been excluded? Is a gene-disrupting translocation in DISC1 merely a red herring for causal variation elsewhere in the genome? For example, some translocations are not copy number neutral, causal genetic variation in the vicinity of the breakpoints could be 'hitchhiking' due to limited recombination within the pedigree, and the disease status could result from an entirely distinctive mechanism from what has been stated.

Finally, causal environmental effects can also cluster in extended pedigrees. The high prevalences of conduct disorder and recurrent MDD in this pedigree are notable. As these can emerge from the 'matrix of disadvantage', it is possible that nongenetic effects have an etiological role in this pedigree.

\section{GENETIC FINDINGS IN OTHER SAMPLES}

To the best of my knowledge, $t(1 ; 11)(q 42.1 ; q 14.3)$ is private to this Scottish pedigree and has never been reported elsewhere. I am aware of no copy number variants in the DISC1 region that are significantly more common in cases with schizophrenia, bipolar disorder or autism in comparison to controls. ${ }^{24}$

Genome-wide linkage meta-analyses for schizophrenia and bipolar disorder do not provide support for DISC1 or for the chr11 side of the translocation. ${ }^{26,27}$

For common genetic variation, candidate gene studies have reported genetic associations with various psychiatric disorders in DISC1. However, these small studies are known to have issues with quality control. The largest and most comprehensive studies show no common single-nucleotide polymorphism (SNP) association signal in the DISC1 region. The PGC (Psychiatric Genomics Consortium) schizophrenia GWAS (genome-wide association study) mega-analysis (9394 cases and 12462 controls) had a minimum $P=0.02$ in DISC1, a level of significance about 1 million times larger than that required for genome-wide significance. ${ }^{28} \mathrm{~A}$ separate meta-analysis of DISC1 variants from 10 candidate gene studies and 3 GWAS (11626 schizophrenia cases and 15237 controls) found no significant associations even at a liberal genewise significance level. ${ }^{29}$ Similarly, a yet larger GWAS shows no DISC1 evidence (Sullivan, submitted). There are also no notable findings on the chr11 side of the translocation.

Some papers have hypothesized that the effects of DISC1 are pleomorphic in the sense of predisposing to multiple psychiatric disorders. The PGC cross-disorders group has conducted an integrated GWAS mega-analysis of 61220 subjects, including cases with schizophrenia, bipolar disorder, MDD, autism and attention deficit-hyperactivity disorder. ${ }^{25}$ This analysis directly and systematically evaluated the pleomorphic effects of common genetic variation in DISC1, effectively testing whether any common SNP was associated with more than one disorder. There were no notable associations in the DISC1 region (minimum $P=0.02$, a million times larger than that required for genome-wide significance).

There are few published resequencing studies of DISC1, and larger and more comprehensive studies are in progress. To date, the largest published study of rare exonic variation was negative (discovery in 727 schizophrenia cases and 733 controls, replication in 2191 cases and 2659 controls). ${ }^{10}$ Some smaller studies have claimed association although replication efforts were absent or negative. The strong assertion that $2 \%$ of the attributable risk for schizophrenia was due to rare DISC1 variants ${ }^{30}$ has not been replicated.

\section{THE GENETIC EVIDENCE FOR DISC1 IS NOT STRONG}

Confident associations in human genetics require evidence of statistical association beyond chance and replication in multiple independent samples. ${ }^{31}$ Moreover, we have come to expect exceptional quality control and vigorous efforts to understand the impact of many different types of bias. In my view, the central goal of psychiatric genetics is now the identification of high-confidence associations and not the potential confusion engendered by lists of 'intriguing' findings.

The published genetic evidence for an association of DISC1 with schizophrenia does not meet a high standard. The genetic evidence is limited solely to cytogenetic abnormalities within a single pedigree. There is no independent line of genetic evidence (for example, structural variation in other pedigrees, evidence for increased exonic deleterious mutations in cases, or common variant associations). The apparent absence of autism, mental 
retardation and epilepsy, and the presence of recurrent MDD and conduct disorder in this pedigree is perplexing and atypical.

It is certainly possible that the outstanding questions in Table 1 are readily addressed or have already been answered via analyses, of which I am unaware. However, one cannot escape the conclusion that the genetic findings for DISC1 do not now meet community standards in human genetics. DISC1 stands apart: the genetic evidence in support of other rare variants of strong effect have increased in the past decade, whereas the genetic evidence for DISC1 has not. ${ }^{24}$

What about biology? DISC1 proponents argue that its fascinating roles in the development and function of the brain trump the genetic findings. This argument is not accepted in mainstream human genetics: biology does not have a role in establishing a genetic association (but only later in understanding its role). Invoking biology to cover up deficiencies in the genetic evidence is a slippery slope. Most genes have a direct or indirect role in central nervous system biology and any integrative scientist worth his or her salt could make an 'intriguing' case for a large fraction of human genes. To connect DISC1 to any psychiatric disorder requires iron-clad genetic associations, which are currently lacking.

Names are powerful things, and, at present, one could reasonably posit that 'disrupted in schizophrenia' is a misnomer and prone to misinterpretation. The official HUGO gene name unmistakably but incorrectly implies a highly certain role in the etiology of schizophrenia. Unless the genetic evidence improves in the near future, wouldn't it be be scientifically responsible to change the name of DISC1 to a more neutral descriptor?

\section{CONFLICT OF INTEREST}

Dr Sullivan was a member of the SAB of Expression Analysis (Durham, NC)

PF Sullivan

Departments of Genetics and Psychiatry, Center for Psychiatric Genomics, University of North Carolina at Chapel Hill, Chapel Hill, NC, USA E-mail: pfsulliv@med.unc.edu

\section{REFERENCES}

1 Neale BM, Kou Y, Liu L, Ma'ayan A, Samocha KE, Sabo A et al. Nature 2012; 485 242-245.

2 O'Roak BJ, Vives L, Girirajan S, Karakoc E, Krumm N, Coe BP et al. Nature 2012; 485 246-250.
3 Sanders SJ, Murtha MT, Gupta AR, Murdoch JD, Raubeson MJ, Willsey AJ et al. Nature 2012; 485: 237-241.

4 Need AC, McEvoy JP, Gennarelli M, Heinzen EL, Ge D, Maia JM et al. Am J Hum Gen 2012; 91: 303-312.

5 Xu B, lonita-Laza I, Roos JL, Boone B, Woodrick S, Sun Y et al. Nat Genet 2012; 44 1365-1369.

6 MacArthur DG, Balasubramanian S, Frankish A, Huang N, Morris J, Walter K et al. Science 2012; 335: 823-828.

7 Millar JK, Wilson-Annan JC, Anderson S, Christie S, Taylor MS, Semple CA et al. Hum Mol Genet 2000; 9: 1415-1423.

8 St Clair D, Blackwood D, Muir W, Carothers A, Walker M, Spowart G et al. Lancet 1990; 336: 13-16.

9 Sullivan PF, Lin D, Tzeng JY, van den Oord EJCG, Perkins D, Stroup TS et al. Mol Psychiatry 2008; 13: 570-584.

10 Crowley J, Hilliard C, Kim Y, Morgan M, Lewis L, Muzny D et al. Mol Psychiatry 2012; 18: $138-140$.

11 Porteous DJ, Millar JK, Brandon NJ, Sawa A. Trends Mol Med 2011; 17: 699-706.

12 Porteous D. Curr Opin Genet Dev 2008; 18: 229-234.

13 Insel TR. Nature 2010; 468: 187-193.

14 Ming GL, Song H. Cell 2009; 136: 990-992.

15 Duan X, Chang JH, Ge S, Faulkner RL, Kim JY, Kitabatake Y et al. Cell 2007; 130 $1146-1158$.

16 Park YU, Jeong J, Lee H, Mun JY, Kim JH, Lee JS et al. Proc Natl Acad Sci USA 2010; 107: 17785-17790.

17 Ishizuka K, Kamiya A, Oh EC, Kanki H, Seshadri S, Robinson JF et al. Nature 2011; 473: 92-96.

18 Korth C. Prion 2012; 6: 134-141.

19 Jacobs PA, Brunton M, Frackiewicz A, Newton M, Cook PJL, Robson ER. Ann Hum Genet 1970; 33: 325-336.

20 Blackwood DH, Fordyce A, Walker MT, St Clair DM, Porteous DJ, Muir WJ. Am J Hum Genet 2001; 69: 428-433.

21 Kelsoe JR, Ginns El, Egeland JA, Gerhard DS, Goldstein AM, Bale SJ et al. Nature 1989; 342: 238-243.

22 Sullivan PF, Neale MC, Kendler KS. Am J Psychiatry 2000; 157: 1552-1562.

23 Major Depressive Disorder Working Group of the Psychiatric GWAS Consortium. Mol Psychiatry 2013; 18: 497-511.

24 Sullivan PF, Daly MJ, O’Donovan M. Nat Rev Genet 2012; 13: 537-551.

25 Identification of risk loci with shared effects on five major psychiatric disorders: a genome-wide analysis. Lancet; e-pub ahead of print 27 February 2013.

$26 \mathrm{Ng} \mathrm{MY}$, Levinson DF, Faraone SV, Suarez BK, DeLisi LE, Arinami T et al. Mol Psychiatry 2009; 14: 774-785.

27 McQueen MB, Devlin B, Faraone SV, Nimgaonkar VL, Sklar P, Smoller JW et al. Am J Hum Genet 2005; 77: 582-595.

28 Schizophrenia Psychiatric Genome-Wide Association Study Consortium. Nat Genet 2011; 43: 969-976.

29 Mathieson I, Munafo MR, Flint J. Mol Psychiatry 2012; 17: 634-641.

30 Song W, Li W, Feng J, Heston LL, Scaringe WA, Sommer SS. Biochem Biophys Res Commun 2008; 367: 700-706.

31 Chanock SJ, Manolio T, Boehnke M, Boerwinkle E, Hunter DJ, Thomas G et al. Nature 2007; 447: 655-660. 